Running head: TRANS-CONTEXTUAL MODEL INTERVENTION

Effects of a School-based Intervention on Motivation for Out-of-school Physical Activity

\title{
Participation
}

\author{
Vassilis Barkoukis ${ }^{1}$, Nikos L. D. Chatzisarantis², \& Martin Hagger ${ }^{3,4}$ \\ ${ }^{1}$ Aristotle University of Thessaloniki \\ ${ }^{2}$ Curtin University \\ 3University of California, Merced \\ 4University of Jyväskylä
}

Full citation: Barkoukis, V., Chatzisarantis, N. L. D., \& Hagger, M. S. (2020). Effects of a school-based intervention on motivation for out-of-school physical activity participation. Research Quarterly for Exercise and Sport. https://doi.org/10.1080/02701367.2020.1751029 


\section{Effects of a School-based Intervention on Motivation for Out-of-school Physical Activity}

\section{Participation}

\section{Abstract}

Purpose: We tested effects of an autonomy-supportive intervention in physical education (PE) on high-

school students' autonomous motivation in PE, and their autonomous motivation, intentions, and physical activity (PA) behavior in a leisure-time guided by the trans-contextual model.

Method: PE classes in two schools were assigned to receive either an autonomy-supportive intervention and or a control intervention via random allocation by school. The PE teacher of the school assigned to the autonomy-supportive intervention was trained to provide autonomy support while the

$P E$ teacher of the school assigned to the control intervention received no training. Students $(N=256)$ in all classes completed measures of perceived teacher autonomy support, autonomous motivation in PE and leisure-time, and beliefs, intentions, and PA in leisure-time before and immediately after the intervention.

Results: Results revealed direct effects of the autonomy-supportive intervention on changes in perceived autonomy support. However, there were no direct intervention effects on change in intentions and PA behavior. The intervention also had indirect effects on changes in autonomous motivation in PE and leisure time. Additionally, change in perceived autonomy support had direct effects on change in autonomous motivation in PE, and indirect effects on change in leisure-time autonomous motivation. Changes in autonomous motivation in leisure-time had direct effects on changes in beliefs and indirect effects on changes in intentions and PA behavior through changes in beliefs.

Conclusion: The study provides valuable information on the effect of autonomous supportive climate on students' beliefs toward PA in PE lessons and in their leisure time outside of school.

24 Keywords: trans-contextual model, self-determination theory, theory of planned behavior, selfregulation. 
Evidence suggests that physical inactivity has deleterious effects on the physical and mental health in young people and contributes to increased risk of chronic disease. For example, low levels of physical activity has been associated with overweight and obesity and higher risk for cardiovascular disease in school-aged children (Carson et al., 2016; Kurdaningsih, Sudargo, \& Lusmilasari, 2017). Furthermore, low levels of physical activity has been shown to be related to depressive symptoms, psychological distress, low self-esteem, hyperactivity and attention problems, anti-social behavior, and impaired psychological well-being and perceived quality of life (Carson et al., 2016; Hoare, Milton, Foster, \& Allender, 2016; Suchert, Hanewinkel, \& Isensee, 2015). In contrast, regular participation in physical activity in young people is associated with reduced risk of illness, and positive mental health outcomes (Biddle, Ciaccioni, Thomas, \& Vergeer, 2019; Ekelund et al., 2009). These benefits notwithstanding, there is consistent evidence that children and adolescents in many nations do not participate in sufficient physical activity confer these health benefits and reduce disease risk.

Governments and health departments have therefore produced guidelines and recommendations on the appropriate levels of physical activity for good health in young people, and developed strategy documents and interventions to promote physical activity (Breda et al., 2018). Physical education (PE) stands as a useful existing network that can be utilized to deliver interventions aimed at fostering regular participation in physical activity in children and adolescents

44 (Cooper et al., 2016). This has led researchers to explore potential strategies on how to promote increased physical activity in PE students, an endeavor that necessitates an understanding of how contextual factors in PE can foster students motivation toward physical activty. One perspective has been to study how the behaviors displayed by social agents (e.g., teachers, parents, peers) in social contexts can promote motivation and behavior toward activities in class. With respect to PE lessons, an autonomy-supportive environment has been shown to result in adaptive responses in students related to the lesson itself such as vitality, enjoyment, effort, and reduced anxiety (Liukkonen, Barkoukis, Watt, 
been focused on the role of school PE in promoting students' out-of-school participation in physical activity, an important priority for PE teachers, health educators, and curriculum developers (Klein \& Hardmann, 2007).

There is, however, growing evidence that an autonomy-supportive environment in PE may promote out-of-school physical activity (Hagger \& Chatzisarantis, 2016; Hagger, Chatzisarantis, Culverhouse, \& Biddle, 2003). Specifically, research has focused on identifying how promoting students' motivation toward activities in PE may also affect their motivation toward, and actual participation in, physical activity outside of school. The trans-contextual model (TCM; Hagger et al., 2003) was developed for this purpose, and aims to describe the process by which support for autonomous motivation in school influences students' participation in related activities outside school.

The model integrates core constructs and processes from self-determination theory (Deci \& Ryan, 1985, 2002), the hierarchical model of intrinsic and extrinsic motivation (Vallerand, 1997, 2007; Vallerand \& Ratelle, 2002), and theory of planned behavior (Ajzen, 1985, 1991, 2002). Selfdetermination theory (Deci \& Ryan, 1985) specifies how the social environment (i.e., motivational climate) in educational settings relates to motivation and, importantly persistence on tasks (see Hagger, Hardcastle, Chater, Mallett, Pal, \& Chatzisarantis, 2014). Vallerand's $(1997,2007)$ hierarchical model describes the process by which motivation is transferred between different contexts. The theory of planned behavior (Ajzen, 1985) outlines the decision making process by which individuals' beliefs and intentions with respect to particular behaviors lead to future behavioral participation. According to the model, an autonomy-supportive environment in school PE will foster students' autonomous motivation in PE which, in turn, will be transferred into autonomous motivation for out-of-school physical activity participation. Autonomous motivation for physical activity participation will influence actual behavior, through the belief-based constructs from the theory of planned behavior (i.e., attitudes, perceived behavioral control, subjective norms and intentions; Chan, Zhang, Lee, \& Hagger, 2020; Hagger et al., 2003). 
The key premises of the model have received substantial empirical support in school PE and leisure-time physical activity (Barkoukis \& Hagger, 2009, 2013; Hagger et al., 2003; González-Cutre et al., 2014a; González-Cutre, Sicilia, Beas-Jimenez, \& Hagger, 2014b; Moreno-Murcia, Hernandez, Pedreno, \& Neipp, 2017; Ntovolis, Barkoukis, Michelinakis, \& Tsorbatzoudis, 2015; Shen, McCaughtry, \& Martin, 2007, 2008). In addition, the cross-cultural invariance and replicability of the TCM in countries with notable cultural differences has been supported (Hagger et al., 2005; Hagger et al., 2009). Furthermore, evidence confirmed the utility of the model beyond PE; in science (Hagger \& Hamilton, 2018), mathematics (Hagger, Sultan, Hardcastle, \& Chatzisarantis, 2015; Hagger et al., 2016), and for after school learning (Chan, et al., 2015). Research has also supported the predictions of the model beyond school PE, supporting the generalizability of its predictions (e.g., Chan et al., 2011, 2015; Hagger et al., 2016). A recent meta-analysis of studies applying the model in PE contexts also provides converging evidence supporting model predictions (Hagger \& Chatzisarantis, 2016).

One of the key propositions of the TCM is that students' autonomous motivation will transfer across contexts and affect physical activity participation outside of school. It stands to reason, that fostering autonomous motivation in PE may be effective in promoting autonomous motivation toward, and actual participation in, physical activities in leisure time. PE may therefore serve as an opportune environment to administer interventions that target change in autonomous motivation that may have ramification beyond school. Research has demonstrated that social agents such as teachers can be effective in promoting greater autonomous motivation through the display of autonomy-supportive behaviors and autonomy-supportive interpersonal communications with students in lessons.

Prior research has consistently supported the positive effect of autonomy supportive environments on students' adaptive responses with respect to in- and out-of school cognition, affect, and behavior (Chatzisarantis, Hagger, \& Brickell, 2008; Hagger \& Chatzisarantis, 2016; Hastie, Rudisill and Wadsworth, 2013; Su \& Reeve, 2011). Hence, in order to achieve positive outcomes from participation in PE lessons emphasis should be placed in the adoption of an autonomy supportive 
motivational climate. Such a climate adopts the students' perspective, allows students to express their thoughts and feelings, and promotes students' self-regulation (Reeve, 2009). In an autonomysupportive motivational climate, teachers nurture students' inner motivational resources (e.g., interests, preferences, psychological needs), provide explanatory rationales (e.g., articulate the sometimes hidden usefulness underlying a teacher's request), rely on noncontrolling language (e.g., informational communications that help students diagnose and solve their motivational problems), display patience to allow students the time they need for self-paced learning to occur (e.g., allow time for students to work in their own way), acknowledge and accept students' expressions of negative affect (e.g., treat students' complaints as valid reactions to imposed demands and structures), and engender students'

111 sense of choice over their behavior (Reeve, 2009, 2016; Reeve \& Jang, 2006; Teixeira et al., 2020).

112 Experimental studies in educational contexts have confirmed the positive effect of an autonomy supportive climate on students' responses (Su \& Reeve, 2011). Furthermore, several intervention

114 studies have demonstrated that programs that train teachers to be more autonomy supportive lead to

115 greater use of autonomy-supportive strategies in the classroom (Cheon, Reeve, \& Moon, 2012;

116 McLachlan \& Hagger, 2010), and adaptive educational outcomes in students including higher levels of

117 autonomous motivation, need satisfaction, future intentions classroom engagement, and skill

118 development (Cheon et al., 2012; Mandigo, Holt, Anderson, \& Shepard, 2008; Murcia, Lacarcel, \&

119 Alvarez, 2010; Perlman, 2010; Tessier, Sarrazin, \& Ntoumanis, 2010). environments in PE. Consistent with this research, the TCM has been suggested as a basis for PE-

122 based interventions (Hagger \& Chatzisarantis, 2016). However, to date, structured interventions using

123 autonomy-supportive teaching strategies in high school PE to promote physical activity outside of 124 school are scarce. Furthermore, with some notable exceptions (e.g., Cheon et al., 2012) all the above mentioned interventions measured outcomes solely in the school context, whereas there is a lack of 
127 school. Furthermore, drawing from the TCM, there is relatively little evidence examining the processes

128 by which school-based autonomy supportive interventions based on self-determination theory relate to

129 physical activity participation outside of school. In particular, there is a dearth of studies examining

130 theory-based motivational and social cognition mediators of the effects of such interventions on leisure-

131 time physical activity in young people. The present study applied the TCM to addess this evidence gap

132 by investigating the effect of an autonomy-supportive school-based intervention on high school

133 students' motivation and beliefs toward, and actual participation in, leisure-time physical activity.

134 Specifically, the study aims to advance knowledge by demonstrating the processes by which an

135 intervention promoting use of autonomy-supportive behaviors in PE teachers relates to out-of-school

136 physical activity behavior in high school students. To date, research on the TCM has been largely

137 confined to correlational, longitudinal and prospective studies, with virtually no data on whether model

138 effects are supported when key constructs, namely perceived autonomy support, and autonomous

139 motivation in PE, are changed through intervention. The current research will, therefore, provide

140 evidence that the key constructs of the model can be changed through intervention, and mediate

141 effects of the intervention on students' out-of-school physical activity participation. In doing so, it may

142 signpost a potentially effective strategy by which PE teachers can support autonomous motivation

143 toward in-school and out-of-school physical activity, which may be useful to promote ongoing physical

144 activity particiption in high school students.

\section{The Present Study}

146 The purpose of the present study was to test the efficacy of a school-based intervention to

147 promote autonomous motivation towards in-class and out-of-school physical activity on key motivational

148 and behavioral variables for physical activity in a leisure-time context based on the motivational

149 sequence specified in the trans-contextual model. We expected the autonomy-supportive intervention

150 to account for changes in psychological variables across baseline prior to the intervention and at follow-

151 up post intervention. We also expected the intervention to account for changes in leisure-time physical 
152 activity behavior at follow-up while controlling for baseline leisure-time physical activity behavior.

153 Overall, we therefore expected changes in the psychological variables specified in the trans-contextual

154 model to mediate the effects of the autonomy-supportive intervention in PE on changes in physical

155 activity intentions and behavior in a leisure-time context. As a consequence, we propose that the

156 intervention effects are modelled as predictors of change in key constructs of the trans-contextual

157 model. Model constructs should, therefore, serve to mediate effects of an autonomy-supportive intervention on participation in leisure-time physical activity.

In terms of specific hypotheses, we expected the intervention to affect students' leisure-time

160 physical activity behavior mediated by changes in the variables specified in motivational sequence of

161 the trans-contextual model. Specifically, we expected the intervention to have effects on changes in

162 perceived autonomy support in $\mathrm{PE}$, and, through this variable, affect changes in autonomous motivation

163 in PE. In addition, we expected trans-contextual effects of changes in autonomous motivation in PE to changes in autonomous motivation in leisure time, consistent with the core hypothesis of the model. We also expected changes in autonomous motivation to impact changes in leisure-time physical activity intentions and actual leisure-time physical activity behavior through the belief-based social cognitive

167 constructs from the theory of planned behavior. Specifically, we expected changes in autonomous motivation in leisure time to be related to changes in intentions via changes in attitudes and perceived behavioral control, and that changes in intentions would predict physical activity behavior. Overall, therefore, we expected statistically significant indirect effects of the intervention and perceived autonomy support in PE on physical activity behavior via the sequence in the model. We expected

172 these proposed effects to hold while controlling for demographic variables (gender, age) and past 173 leisure-time physical activity behavior measured at baseline. 
The study conformed to Aristotle University of Thessaloniki[University identity masked for peer

review] Code of Ethics in Research. Two hundred eighty-one junior high school students took part in

the present study. Of those two hundred fifty-six provided valid data in both measurement points ( $M$ age $=13.46 ; S D=.82$, males $=120$, females $=129$, unreported $=7)$. The remaining students did not

180 complete the questionnaires in one of the measurement points due to absence from the school the data

181 collection days. Students were recruited from two typical co-educational high schools in an urban city in

182 Northern Greece. Both schools were located in the same educational region and the students were of similar socio-demographic background. Students completed a battery of questionnaires on two measurement occasions; before and immediately after the completion of the intervention. The autonomy-supportive training lasted 10 weeks; two lessons per week. In each measurement occasion, a two-wave prospective design, similar to the one typically used in TCM research, was employed. In the

187 first wave of data collection, students completed the measures pertaining to the TPB variables, motivational regulations in leisure-time, and past behavior. In the second wave of data collection, conducted three weeks after the first wave, measures of perceived autonomy support, motivational regulations in PE, and self-reported physical activity behavior were administered. Before questionnaire administration permission from school principals and parents was obtained. Parents received a pre-

192 print form describing the purpose of the study; parents who did not wish their child to complete the questionnaire or take part in the intervention should sign the form and return it to school. No signed forms were returned. Students were informed that they will participate in a survey on students' beliefs about the PE lessons. They were not informed about participation in an intervention study in order to

196 minimize potential Hawthorne-type effects (i.e., modification of behavior as a result of the awareness of

197 being observed; McCarney et al., 2007). The questionnaires were completed in quiet classroom conditions supervised by a trained research associate and without the presence of their PE teacher.

199 Students were informed that the questionnaire was anonymous and were reassured of the confidentiality of their responses and that they would be used solely for research purposes. Students 
were matched across waves and measurement points based on their class, gender and date of birth.

The study was carried out the spring semester of the 2014-2015 academic season. The timeline of the study design and data collection is presented in Figure 1.

\section{Measures}

A battery of measures was used to assess perceived autonomy support, motivational regulations in PE and in leisure-time, and students' social cognition towards leisure-time physical activity. All scales have been used previously with Greek students and demonstrated adequate psychometric properties.

Perceived autonomy support. Students' perceptions of teacher-initiated support were measured with Perceived Autonomy Support Scale for Exercise Settings (PASSES; Hagger et al. 2007). The PASSES comprises 12 items representing respective autonomy supportive behaviors during PE classes (example item 'I feel that my PE teacher provides me with choices, options, and opportunities to do active sports and/or vigorous exercise'). Hagger et al. (2007) provided evidence on

214 the validity of the scale and Barkoukis and Hagger (2013) used this scale with Greek high school students. Students responded on a 7-point scales ranging from 1 ('strongly disagree') to 7 ('strongly agree'). The scale demonstrated adequate internal consistency.

218 \& Connell, 1989) was used to assess students' motivational regulations in PE. The scale includes four

219 motivational regulations, two autonomous: intrinsic motivation (e.g. "...it is fun") and identified regulation

220 (e.g. "...I value PE"), and two controlled: introjected regulation (e.g. "...I will feel ashamed if I do not do 221 PE”), and external regulation (e.g. '... important others want me to do PE”). Participants responded to 222 the stem question 'I participate in PE because...' on a 4-point Likert scale ranging from 1 ('not true at all') to 4 ('very true').

Motivational regulations in leisure-time. Students' motivational regulations in leisure time

225 physical activity were assessed with the Behavioral Regulations in Exercise Questionnaire (Mullen 
226 Markland, \& Ingledew, 1997). Participants responded to the stem question 'Why do you participate in active sports and/or vigorous physical activities in your leisure time?' followed by 15 items measuring four motivational regulations; intrinsic motivation (e.g. '... because it is fun'), identified regulation (e.g. '...because it is important to make the effort'), introjected regulation (e.g. ' ...because I will feel guilty if I do not'), and external regulation (e.g. ' ... because others say I should'). Responses were anchored on a 7-point Likert scale ranging from 1 ('not true at all') to 7 ('very true'). In order to reduce the number of constructs in subsequent analyses and develop more parsimonious model a relative autonomy index (RAI) was calculated for both the PE and LTPA measures of motivation based on Vallerand's (2007) recommendations (i.e., $2 \mathrm{x}$ intrinsic motivation + identified regulation - introjected regulation $-2 \mathrm{x}$ external regulation). The RAI was used in all subsequent analyses.

Theory of Planned Behavior variables. The TPB variables in the present study were assessed based on the recommendations of Ajzen (2002) and previous research with the TCM (e.g., Barkoukis \& Hagger, 2013). The measure of attitudes included five semantic differential scales with the bipolar adjectives: bad-good, harmful-beneficial, not enjoyable-enjoyable, useful-useless and boringinteresting. Students responded to the stem question: 'Participating in active sports and/or vigorous physical activities during my leisure-time in the next five weeks is...' and responses were coded on a 7point scale. Subjective norms were assessed with two items (e.g., 'People important to me think that I should do active sports and/or vigorous physical activities during my leisure-time in the next 5 weeks') with students recording their responses on a 7-point scale ranging from 1 ('strongly disagree') to 7

245 ('strongly agree'). PBC was measured with three items (e.g., "I feel in complete control over whether I

246 do active sports and/or vigorous physical activities in my leisure-time in the next 5 weeks") with

247 responses anchored on a 7-point Likert scale ranging from 1 ('no control') to 7 ('complete control'). Intentions were assessed with three items (e.g., "I intend to do active sports and/or vigorous physical activities during my leisure-time in the next 5 weeks...") rated on seven-point scales anchored by 1

250 ("strongly disagree") to 7 ("strongly agree"). 
Questionnaire was used to measure students' self-reported leisure-time physical activity participation.

Participants then were asked to record their 5-week physical activity participation during their leisuretime on two items (e.g., "In the course of the past five weeks, how often have you participated in vigorous physical activities for 20 minutes at a time?") using six-point Likert scales ranging from 1 ('never') to 6 ('everyday').

Past behavior. A single-item used in past TCM research (Barkoukis \& Hagger, 2013; Hagger et al. 2003) was used to measure participants' past physical activity behavior (i.e., 'In the course of the past six months, how often, on average, have you participated in vigorous physical activities for 20 minutes at a time?'). Participants recorded their physical activity participation on a 6-point Likert scale ranging from 1 ('not at all') to 6 ('most days per week').

Two schools were randomly assigned to receive the autonomy-support intervention or control intervention. The school assigned to receive the autonomy-supportive intervention comprised five classes of students (total $n=131 ; 63$ males and 61 females, 7 students did not report their gender; $M$ age $=13.26$ years, $S D=.84)$. The school assigned to receive the control intervention also comprised five classes (total $n=125$ students; 57 males and 68 females, $M$ age $=13.65$ years, SD $=.76$ ). The PE teacher in the school assigned to receive the autonomy-supportive intervention (male, 43 years old, 14

271 years of experience in secondary education) attended a series of 3 seminars lasting 1.5 hours each

272 over a period of 2 weeks. The first session included a description of key concepts from selfdetermination theory and their interplay in establishing an adaptive social environment that will result in students adopting adaptive behaviors in the school context (e.g., relations between autonomy supportive climate and intrinsic motivation). The second session included instruction on how to adopt 
276 strategies that promote autonomy in students (e.g., providing choice, adopting active listening,

277 encouraging student-generated questions, allowing students to work independently, providing a

278 meaningful rationale, providing informational feedback, offering hints, responding to student-generated

279 questions). The third session included instruction on strategies on how to avoid fostering a controlling

280 climate (e.g., avoiding use of controlling language (e.g., "should", "must" and "got to", avoiding giving

281 solutions verbatim, avoiding use of directives and commands) (see Reeve \& Jang, 2006; Teixeira et al.,

282 2020). All three sessions were interactive emphasizing discussion of examples from everyday life,

283 demonstrating how to promote interaction with students during lessons, and discussing solutions to

284 existing or anticipated situations during the implementation of the intervention (e.g., number of choices,

285 types of choices in each subject). The PE teacher of the school assigned to receive in the autonomy-

286 supportive intervention was then instructed to apply this training to his regular PE lessons for a period

287 of 10 weeks. For example, during the warm-up phase, the PE teacher offered students choice with

288 respect to the type of warm-up activity or was allowed students to do their own stretching exercises. In

289 addition, the PE teacher was explaining the content of the lesson, providing rationales for the selected

290 activities. In the main part of the lesson, practice and inclusion teaching styles were endorsed allowing

291 students to work at their own pace. Furthermore, on several occasions, students were asked to choose

292 among similar drills to perform, or chose the order of the drills to be performed. Also, depending on the

293 content of the lesson, goal setting was fostered. At the end of the lesson a few minutes were devoted to

294 asking questions to students about their experiences in the lesson (see Table 1 for example strategies).

295 The PE teacher of the school assigned to receive the control intervention (male, 45 years old, 17 years

296 of experience in secondary education) was told that his school was selected to participate in a study

297 investigating the short term effects of PE lessons on students' beliefs about the lesson and received no

298 training on self-determination or promotion of autonomy supportive climate. The PE teacher was asked

299 to teach their normal PE lessons for a period of 10 weeks. An informal discussion with the PE teacher

300 after the completion of the second measurement point revealed that he did not change his teaching 
approach. Lesson content in both schools was guided by the national curriculum for PE (see

Tsorbatzoudis, Grouios, Barkoukis \& Alexandris, 2007). Both teachers taught the same sport activities

(i.e., basketball, football, track and field), and Greek traditional dances, but the order of the subjects or

the specific dances taught and the time devoted to each activity, was slightly different depending on the school facilities, weather, and other conditions.

\section{Data Analysis}

Descriptive statistics and reliability analyses were computed using the psych package (Revelle, package (Rosseel, 2012) in R using a maximum likelihood estimation method ${ }^{1}$. We also estimated

310 bootstrapped standard errors with 1000 replications, consistent with recommendations (Hayes, 2018).

311 Missing data was imputed using the full-information maximum likelihood method (FIML)2. As more than

$31295 \%$ of cases were retained across the baseline and follow-up time points of the intervention, attrition

313 analyses were redundant. All the psychological and behavioral variables in the model were represented

314 as change variables. The change variables were computed as residualized change scores derived from

315 the regression of the follow-up measure of the variable on its baseline value. The hypothesized

316 relations among the variables in the proposed model are summarized in Figure 2. The pattern of

317 proposed effects followed hypotheses derived from the trans-contextual model. The effects of the

318 intervention were tested by predicting leisure-time physical activity at follow-up, the primary dependent

319 variable, changes in intention, and changes in perceived autonomy support by a dichotomous

320 intervention variable coded as $1=$ control intervention and $2=$ autonomy-supportive intervention.

321 Gender, age, and leisure-time physical activity behavior at baseline were included as control variables

322 which predicted all other variables in the model. Age and baseline physical activity behavior, were

323 included as continuous control variables and gender was included as a dichotomous control variable.

\footnotetext{
${ }^{1}$ Data files, analysis scripts, and output from the data analysis including reliability, statistical power, and path analyses are available online: https://osf.io/b4t9c

2Path analyses were conducted without FIML imputation, patterns of effects were unchanged, full results are available online: https://osf.io/b4t9c
} 
324 Adequacy of the hypothesized model was established using the goodness-of-fit chi-square, the comparative fit index (CFI), with values exceeding .95 typically considered appropriate cutoff values for adequate model fit, the standardized root mean squared residuals with a cutoff value of 0.500 taken as indicating a well-fitting model, and the root mean squared error of approximation (RMSEA) and its $90 \%$ confidence intervals $\left(\mathrm{Cl}_{90}\right)$ with a cutoff value equal to or less than .08 and narrow confidence intervals

329 indicative of an adequately-fitting model (Marsh, Hau, \& Wen, 2004). Hypothesized mediation effects were tested by calculating indirect effects with bootstrapped standard errors.

\section{Results}

Descriptive statistics, reliability coefficients ${ }^{3}$, and zero-order correlation coefficients among study variables are reported in Table 2. Reliability coefficients revealed acceptable reliabilities for all constructs at each time point. Exceptions were the autonomous motivation in PE (relative autonomy index) scale at baseline and follow-up and the subjective norm scale at baseline. Standardized parameter estimates for the path analysis among the proposed model constructs are presented in

337 Figure 3. Overall, the model exhibited adequate fit with the data $\left(X^{2}(18)=44.390, p=.001 ; C F I=.956\right.$; SRMSR $=.045 ;$ RMSEA $=.078$, RMSEA Cl $_{90}$ upper limit $=.049$, RMSEA Cl $_{90}$ lower limit $\left.=.107\right)$. In

339 addition, the model accounted for a statistically significant amount of variance in the key dependent 340 variables: follow-up leisure-time physical activity $\left(R^{2}=.451\right)$, changes in intentions $\left(R^{2}=.439\right)$, and 341 changes in perceived autonomy support $\left(R^{2}=.210\right)$. In addition, a posteriori statistical power analysis 342 for the final model was conducted to ensure the final model had the requisite power. Our analysis was 343 based on MacCallum, Browne, and Sugawara's (1996) method based on the RMSEA and implemented 344 using the Webpower tool (Zhang \& Yuan, 2018). Based on input parameters recommended for a 345 conservative estimate $(N=271, H 1$ RMSEA $=.078, H 0$ RMSEA $=.000, d f=18, p=.001)$, the 346 reproduced statistical power was .851.

\footnotetext{
${ }^{3}$ Where possible Omega reliability coefficients (Revelle \& Zinbarg, 2008) were computed. For two-item scales, the Spearman-Brown inter-item correlation was computed. For the relative autonomy index in leisure time at baseline, the Omega reliability calculation did not converge due a non-positive definite matrix, so the standard Cronbach alpha is reported.
} 
Results revealed no statistically significant effects of the intervention on follow-up leisure-time physical activity $(\beta=.049, p=.329)$ and changes in intentions $(\beta=-.024, p=.637)$. Unsurprisingly, there was a significant large-sized effect of the intervention on changes in perceived autonomy support $(\beta=.466, p<.001)$. There were statistically significant direct effects of changes in perceived autonomy support on changes in autonomous motivation in $\mathrm{PE}(\beta=.188, p=.002)$ and changes in autonomous motivation in leisure time $(\beta=.117, p=.044)$, with small effect sizes. There was also a statistically significant direct effect of changes in autonomous motivation in leisure time on changes in PBC $(\beta=$ $.318, p=.025)$ as hypothesized with a small-to-medium effect size. Contrary to hypotheses, a we found a statistically significant negative direct effect of changes in autonomous motivation in leisure time on changes in subjective norms $(\beta=-.386, p<.001)$ with a small-to-medium effect size. Changes in intentions were predicted by changes in PBC $(\beta=.610, p<.001)$ with a medium-to-large effect size, as hypothesized. But there was not effect of changes in attitudes $(\beta=.039, p=.489)$ or subjective norms $(\beta=.106, p=.069)$ on intentions, contrary to hypotheses. Changes in intentions predicted participation in leisure time physical activity $(\beta=.559, p<.001)$ with a medium-to-large effect size. In terms of indirect effects, we found statistically significant indirect effects of the intervention on changes in autonomous motivation in PE via changes in perceived autonomy support $(\beta=.087, p=$ .003) with a small effect size. We also found statistically significant indirect effects of the intervention on changes in autonomous motivation in leisure time via changes in perceived autonomy support and changes in autonomous motivation in $\mathrm{PE}(\beta=.038, p=.005)$ with a small effect size. There was also an indirect effect of the intervention on autonomous motivation in leisure time via changes in perceived

367 autonomy support alone which fell marginally short of the conventional level for statistical significance $368(\beta=.055, p=.052)$, again, with small effect sizes. Together these resulted in a small, statistically significant total indirect effect of the intervention on changes in autonomous motivation in leisure time ( $\beta$ $=.093, p=.004)$. There were, however, no indirect effects of the intervention on changes in intention or physical activity behavior. We also found a statistically significant indirect effect of changes in perceived 
372 autonomy support in PE on changes in autonomous motivation in leisure time through changes in

373 autonomous motivation in $\mathrm{PE}(\beta=.082, p=.004)$ with a small effect size, consistent with hypotheses.

374 There was also a statistically significant indirect effect of changes in autonomous motivation in leisure-

375 time on changes in intentions through changes in perceived behavioral control $(\beta=.194, p=.036)$, with

376 a small-to-medium effect size, as predicted, but not through attitudes and subjective norms leading us

377 to reject these hypotheses. There was a statistically significant indirect effect of changes in perceived

378 behavioral control $(\beta=.342, p<.001)$ with a small-to-medium effect size via changes in intentions on leisure time physical activity as hypothesized, but not for attitudes or subjective norms, leading us to reject this hypothesis. There was also a statistically significant indirect effects of changes in autonomous motivation in leisure-time on leisure time physical activity via changes in perceived behavioral control and intentions $(\beta=.109, p=.048)$ with a small effect size.

\section{Discussion}

The purpose of the present study was to test the effectiveness of a school-based intervention to promote an autonomy-supportive motivational climate in PE in promoting intentions toward, and actual participation in, leisure-time physical activity behavior outside of school. The study was guided by the trans-contextual model which identified the psychological processes by which the intervention was expected exert its effects on out-of-school leisure-time physical activity intentions and behavior. In the study, students in classes in two schools received either an autonomy-supportive intervention or a control intervention with random assignment at the school level. The PE teacher of the school assigned to receive the autonomy-supportive intervention received a three-seminar interactive program providing

392 them with training to promote autonomy support to students in PE lessons. The teacher in the school

393 assigned to receive the control intervention did not receive the training program. The intervention lasted

39410 weeks. During the intervention, each teacher taught their normal PE lessons concurrent to the training. Results indicated that students in the school allocated to receive the autonomy-supportive

396 intervention reported significantly greater levels of perceived autonomy support and, indirectly, 
autonomous motivation in PE, and autonomous motivation outside of school at post-intervention follow-

up. However, there were no direct effects of the intervention on students' intentions to engage in perceived autonomy support on autonomous motivation in PE and leisure time, and on intentions to be physically active through autonomous motivation and perceived behavioral control, but these variables did not transmit the effects of the intervention on actual physical activity participation. autonomy support from teachers in PE contexts predicted students' autonomous motivation in PE, autonomous motivation toward engaging in physical activity in leisure time, and students' intentions to engage in leisure-time physical activity. Results are, therefore, largely consistent with previous prospective tests of the model (Barkoukis \& Hagger, 2013; Hagger et al., 2003, 2005; 2009), along with review (Barkoukis \& Hagger, 2012; Hagger \& Chatzisarantis, 2012) and meta-analytic (Hagger \&

411 Chatzisarantis, 2016) evidence supporting the major premises of the model, including the indirect

412 effects across contexts, which are central to the model. PE teachers are encouraged to use autonomy

413 supportive practices such as providing rationales, allowing students to work on their own pace,

414 providing opportunities for interaction with the students and being responsive to their question, avoiding use of controlling language (e.g., "should", "must" and "got to"), avoiding giving solutions verbatim, avoiding use of directives and commands, and avoiding asking controlling questions (see Reeve, 2016;

417 Reeve \& Jang, 2006). As our study showed, these practices can increase students' perceptions of an

418 autonomy supportive motivational climate and influence, therefore, their beliefs towards PE lesson and 419 leisure-time physical activity. 
422 effectiveness of model in promoting change in perceived autonomy support and autonomous motivation, but not intentions and physical activity behavior outside of school. Given the consistency and strength of the trans-contextual effects found in previous research of perceived autonomy support and autonomous motivation in a PE context on autonomous motivation, intentions, and actual physical activity engagement in a leisure-time context, it stands to reason that an intervention that evokes change in the constructs in PE may lead to trans-contextual effects on motivation and behavior outside of school. The current intervention was specifically designed to change the key PE variables that have been shown to relate to out-of-school behavior using an intervention that promotes an autonomysupportive motivational climate, and compare effects with an appropriate "no training" comparison (c.f.,

431 Cheon et al., 2012; Reeve \& Jang, 2006). While the autonomy-supportive intervention was effective in promoting greater perceived autonomy support and autonomous motivation, consistent with previous research on autonomy-supportive climates (e.g., Cheon et al., 2012), and in changing autonomous motivation in an out-of-school leisure-time physical activity context, the effects were relatively weak and were not transmitted to intentions to engage in, and actual participation in, subsequent leisure-time physical activity behavior. This is inconsistent with a previous intervention deriving its hypotheses from the trans-contextual model, which showed significant effects of a school-based intervention on leisuretime physical activity behavior through the mediators proposed in the model (Chatzisarantis \& Hagger, 2009).

Possible reasons why the current intervention had little effect may be that the changes made to

441 the practice of the teacher may have been insufficiently strong to lead to changes in out-of-school

442 physical activity, despite changes in out-of-school autonomous motivation. In other words, the 443 intervention effects were not sufficiently powerful to have an effect on physical activity behavior in 444 leisure time, an activity that is distal to PE. Instead, physical activity likely to be subject to more 445 proximal factors that influence behavioral engagement including motivational and social cognition 446 constructs like perceived autonomy support for physical activity outside of school from parents and 
447 peers, attitudes and beliefs toward physical activity, and self-efficacy and socio-ecological factors like

448 access to facilities and opportunities to act (e.g., González-Cutre et al., 2014b; Olson, Ireland, March,

449 Biddle, \& Hagger, 2019). Another possible reason is that the changes in PE teacher's practice were

450 perhaps modest or not maintained in the current intervention relative to others. This may have been

451 because teacher's training was relatively brief and of low intensiveness relative to previous

452 interventions. For example, Chatzisarantis and Hagger's autonomy-support training program involved training of teachers for a total of 3 days training with 3-hour sessions and over a period of 5 weeks,

similar to other autonomy-support interventions (e.g., Cheon et al., 2012; Polet et al., 2019). In contrast, the current intervention was less intense with a total of 4.5 hours training over a period of a week. The intensiveness and duration of training is likely to be a moderator of the effectiveness of autonomy supportive interventions, particularly the strength of the effects and the likelihood that it will pervade into other contexts. Based on meta-analytic evidence that minimal autonomy support interventions with brief training experience are the least effective in changing autonomous motivation and behavior (Su \& Reeve, 2011), there is strong advocacy that autonomy-support interventions to change the behavior of social agents creating motivational climates in educational settings involves multiple exposures over a period of six weeks with reinforcement and feedback on autonomy supportive techniques (Cheon et al., 463 2012). The relatively brief nature of the teachers' training, therefore, may explain the lack of effects on actual behavior across contexts in the current study.

The current study has numerous strengths including: targeting an important research question,

467 namely, whether fostering students' autonomous motivation in PE will lead to physical activity

468 motivation and behavior outside of school in a leisure time context; the adoption of an appropriate integrated multi-theory model to guide the development of the intervention and map the processes by which the intervention exerts effects across PE and leisure-time contexts; the recruitment of a large 471 sample of students and recruiting PE teachers willing to make changes to their interpersonal style and 
472 motivational climate in lessons; the use of previously-validated techniques to foster autonomy-

473 supportive styles in teachers; the use of an intervention design with autonomy-support and control

474 intervention conditions with random allocation by school; and the use of validated measures of

475 psychological mediators of intervention effects and path analytic models using residualized change

476 scores that enabled a test of mediation effects of the model specified a priori while incorporating

477 change across time of measurement.

478 There are, or course, limitations that must be acknowledged. The primary limitation is the

relatively brief, minimal nature of the teacher training relative to other intervention programs adopted in previous studies (e.g., Chatzisarantis \& Hagger, 2009; Cheon et al., 2012), an issue to which we alluded to in our previous discussion. A further limitation is the adoption of a self-report measure of physical activity behavior. Although our measure has demonstrated statistically significant correlations against more objective measures of physical activity in previous studies, there is still considerable potential for response bias introducing substantive measurement error into the current analysis. We also did not account for the potential of clustering of students within classes within the two schools. While there is potential for there to be higher likelihood of similar responding to measures withinclasses rather than between classes, the current study was underpowered to estimate a multilevel model that tested within-class variation alongside between-class variation. However, given that the number of classes was small $(n=5)$ and all classes were taught by the same teacher, we expect that the within-class variability component would have been relatively modest. However, conducting an autonomy-support intervention that enables analyses that account for within-participants effects would

492 be an important avenue for future research. It is also important to note that not all scales for the 493 measures used in the current study exhibited acceptable reliability. Specifically, the autonomous motivation in PE scale at baseline and follow-up and the subjective norm scale at baseline were below acceptable cutoff values. While the current path analytic models aimed to correct for error in prediction, 
497 not able to be estimated. Current findings should therefore be interpreted with the compromised

498 reliability in mind, which has the potential to inflate or suppress model relations involving these

499 constructs. A final limitation is that we did not provide a formal evaluation of the extent to which the PE

500 teacher receiving the autonomy-supportive training and the teacher that received no training differed in

501 the autonomy-supportive behaviors they adopted in their lessons before and after the intervention. This

502 means that a formal evaluation of the fidelity of the intervention, i.e. whether participating teachers had

503 actually followed the protocol and resulted in actual changes in their behavior could not be conducted.

504 This would make the current study much stronger by providing evidence that the intervention led to

505 changes in key behaviors expected to foster autonomous motivation in students. Future research

506 needs to adopt autonomy supportive training programs of extended duration to promote strong effects,

507 use validated, objective measures of leisure-time physical activity such as accelerometers, and include

508 formal tests of intervention fidelity such as observation of teachers' behavior during lessons for key

509 autonomy-supportive behaviors. 
TRANS-CONTEXTUAL MODEL INTERVENTION

References

512 Ajzen, I. (1985). From intentions to actions: A theory of planned behavior. In J. Kuhl \& J. Beckmann

513 (Eds.), Action-control: From cognition to behavior (pp. 11-39). Heidelberg: Springer.

514 Ajzen, I. (1991). The theory of planned behavior. Organizational Behavior \& Human Decision Processes, 50, 179-211. doi: 10.1016/0749-5978(91)90020-T

516 Ajzen, I. (2002). Residual effects of past on later behavior: Habituation and reasoned action perspectives. Personality and Social Psychology Review, 6, 107-122. doi: 10.1207/S15327957PSPR0602_02

Barkoukis V. \& Hagger, M. S. (2009). A test of the trans-contextual model of motivation in Greek high school pupils. Journal of Sport Behavior, 32, 1-23.

Barkoukis, V. \& Hagger, M. S. (2012). A trans-contextual model of motivation in physical education. In J. N. Franco and A. E. Svensgaard (Eds.) Handbook on Psychology of Motivation: New Research (pp. 169-186). New York, NOVA Science Publishers.

Barkoukis, V., \& Hagger, M. S. (2013). The trans-contextual model: perceived learning and performance motivational climates as analogues of perceived autonomy support. European Journal of Psychology of Education, 28, 353-372. doi: 10.1007/s10212-012-0118-5

Biddle, S. J. H., Ciaccioni, S., Thomas, G., \& Vergeer, I. (2019). Physical activity and mental health in children and adolescents: An updated review of reviews and an analysis of causality. Psychology of Sport and Exercise, 42, 146-155. https://doi.org/10.1016/j.psychsport.2018.08.011

Breda, J., Jakovljevic, J., Rathmes, G., Mendes, R., Fontaine, O., Hollmann, S., ... \& Galea, G. (2018). Promoting health-enhancing physical activity in Europe: Current state of surveillance, policy development and implementation. Health Policy, 122(5), 519-527. doi: 10.1016/j.healthpol.2018.01.015 
536

537

538

539

540

541

542

youth: an update. Applied Physiology, Nutrition, and Metabolism, 41(6), S240-S265. doi: 10.1139/apnm-2015-0630

Chan, D. K. C., Dimmock, J. A., Donovan, R. J., Hardcastle, S., Lentillon-Kaestner, V., \& Hagger, M. S. (2015). Self-determined motivation in sport predicts anti-doping motivation and intention: A perspective from the trans-contextual model. Journal of Science and Medicine in Sport, 18(3), 315-322. doi: 10.1016/j.jsams.2014.04.001

Chan, D. K. C., Hagger, M. S., \& Spray, C. (2011). Treatment motivation for rehabilitation after a sport injury: Application of the trans-contextual model. Psychology of Sport and Exercise, 12, 83-92. doi: 10.1016/j.psychsport.2010.08.005

Chan, D. K. C., Yang, S. X., Hamamura, T., Sultan, S., Xing, S., Chatzisarantis, N. L., \& Hagger, M. S. (2015). In-lecture learning motivation predicts students' motivation, intention, and behaviour for after-lecture learning: Examining the trans-contextual model across universities from UK, China, and Pakistan. Motivation and Emotion, 39(6), 908-925. doi: 10.1007/s11031-015-9506-x

Chan, D. K. C., Zhang, L., Lee, A. S. Y., \& Hagger, M. S. (2020). Reciprocal relations between autonomous motivation from self-determination theory and social cognition constructs from the theory of planned behavior: A cross-lagged panel design in sport injury prevention. Psychology of Sport and Exercise, 48, 101660. doi: 10.1016/j.psychsport.2020.101660

Chatzisarantis, N. L. D., \& Hagger, M. S. (2009). Effects of an intervention based on self-determination theory on self-reported leisure-time physical activity participation. Psychology \& Health, 24, 2948. doi:10.1080/08870440701809533

Chatzisarantis, N. L. D., Hagger, M. S., \& Brickell, T. (2008). Using the construct of perceived autonomy support to understand social influence within the theory of planned behavior. Psychology of Sport and Exercise, 9, 27-44. doi: 10.1016/j.psychsport.2006.12.003 
559 Cheon, S. H., Reeve, J., \& Moon, I. (2012). Experimentally based, longitudinally designed, teacherfocused intervention to help physical education teachers be more autonomy supportive toward their students. Journal of Sport \& Exercise Psychology, 34, 365-396. doi: 10.1123/jsep.34.3.365

Cooper, K. H., Greenberg, J. D., Castelli, D. M., Barton, M., Martin, S. B., \& Morrow Jr, J. R. (2016). Implementing policies to enhance physical education and physical activity in schools. Research Quarterly for Exercise and Sport, 87(2), 133-140. doi: 10.1080/02701367.2016.1164009

Deci, E. L., \& Ryan, R. M. (1985). Intrinsic motivation and self-determination in human behavior. Plenum Press. New York.

Deci, E. L., \& Ryan, R. M. (2002). Handbook of self-determination research. Rochester, NY: University of Rochester Press.

Ekelund, U., Anderssen, S., Andersen, L. B., Riddoch, C. J., Sardinha, L. B., Luan, J., . . Brage, S. (2009). Prevalence and correlates of the metabolic syndrome in a population-based sample of European youth. American Journal of Clinical Nutrition, 89, 90-96. https://doi.org/10.3945/ajcn.2008.26649

Godin, G., \& Shephard, R. J. (1985). A simple method to assess exercise behavior in the community. Canadian Journal of Applied Sport Sciences. Journal Canadien des Sciences Appliquees au Sport, 10, 141-146.

González-Cutre, D., Ferriz, R., Beltrán-Carrillo, V. J., Andrés-Fabra, J. A., Montero-Carretero, C., Cervelló, E., \& Moreno-Murcia, J. A. (2014a). Promotion of autonomy for participation in physical activity: A study based on the trans-contextual model of motivation. Educational Psychology,

580 González-Cutre, D., Sicilia, A., Beas-Jiménez, M., \& Hagger, M. S. (2014b). Broadening the trans-contextual model of motivation: A study with Spanish adolescents. Scandinavian Journal of Medicine \& Science in Sports, 24(4), e306-e319. doi: 10.1016/j.paid.2016.06.036 
583

Hagger, M. S., \& Chatzisarantis, N. L. D. (2012). Transferring motivation from educational to extramural contexts: A review of the trans-contextual model. European Journal of Psychology of Education, 27, 195-212. doi:10.1007/s10212-011-0082-5

Hagger, M. S., \& Chatzisarantis, N. L. D. (2016). The trans-contextual model of autonomous motivation in education: Conceptual and empirical issues and meta-analysis. Review of Educational Research, 86, 360-407. doi: 10.3102/0034654315585005

Hagger, M. S., Chatzisarantis, N. L. D., Barkoukis, V., Wang, C. K. J., \& Baranowski, J. (2005). Perceived autonomy support in physical education and leisure-time physical activity: A crosscultural evaluation of the trans-contextual model. Journal of Educational Psychology, 97, 376390. doi: $10.1037 / 0022-0663.97 .3 .376$

Hagger, M. S., Chatzisarantis, N. L. D., Culverhouse, T., \& Biddle, S. J. H. (2003). The processes by which perceived autonomy support in physical education promotes leisure-time physical activity intentions and behavior: A trans-contextual model. Journal of Educational Psychology, 95, 784795. doi: $10.1037 / 0022-0663.95 .4 .784$

Hagger, M. S., Chatzisarantis, N. L. D., Hein, V., Pihu, M., Soós, I., \& Karsai, I. (2007). The perceived autonomy support scale for exercise settings (PASSES): Development, validity, and crosscultural invariance in young people. Psychology of Sport and Exercise, 8, 632-653. doi:10.1016/j.psychsport.2006.09.001

Hagger, M. S., Chatzisarantis, N. L. D., Hein, V., Pihu, M., Soós, I., Karsai, I., Lintunen, T., \& Leemans, S. (2009). Teacher, peer, and parent autonomy support in physical education and leisure-time physical activity: A trans-contextual model of motivation in four nations. Psychology and Health, 24, 689-711. doi: 10.1080/08870440801956192

Hagger, M. S., \& Hamilton, K. (2018). Motivational predictors of students' participation in out-of-school learning activities and academic attainment in science: An application of the trans-contextual 

model using Bayesian path analysis. Learning and Individual Differences, 67, 232-244. doi: 10.1016/j.lindif.2018.09.002

Hagger, M. S., Hardcastle, S. J., Chater, A., Mallett, C., Pal, S., \& Chatzisarantis, N. L. D. (2014). Autonomous and controlled motivational regulations for multiple health-related behaviors: Between- and within-participants analyses. Health Psychology and Behavioral Medicine, 2, 565601. doi: $10.1080 / 21642850.2014 .912945$

Hagger, M. S., Sultan, S., Hardcastle, S. J., \& Chatzisarantis, N. L. (2015). Perceived autonomy support and autonomous motivation toward mathematics activities in educational and out-ofschool contexts is related to mathematics homework behavior and attainment. Contemporary Educational Psychology, 41, 111-123. doi: 10.1016/j.cedpsych.2014.12.002

Hagger, M. S., Sultan, S., Hardcastle, S. J., Reeve, J., Patall, E. A., Fraser, B. J., . . Chatzisarantis, N. L. D. (2016). Applying the trans-contextual model to mathematics activities in the classroom and homework behaviour and attainment. Learning and Individual Differences, 45, 166-175. doi: 10.1016/j.lindif.2015.11.017

Hastie, P. A., Rudisill, M. E., \& Wadsworth, D. D. (2013). Providing students with voice and choice: lessons from intervention research on autonomy-supportive climates in physical education. Sport, Education and Society, 18, 38-56. doi: 10.1080/13573322.2012.701203

Hayes, A. F. (2018). Introduction to mediation, moderation, and conditional process analysis: A regression-based approach (2nd ed.). New York, NY: Guildford Press. and mental health among adolescents: a systematic review. International Journal of Behavioral Nutrition and Physical Activity, 13, 108. doi: 10.1186/s12966-016-0432-4

Klein G., \& Hardman, K. (2007). Physical Education and Sport Education in the European Union. Paris: Editions Revue EP.S. 
631 Kurdaningsih, S. V., Sudargo, T., \& Lusmilasari, L. (2017). Physical activity and sedentary lifestyle towards teenagers' overweight/obesity status. International Journal of Community Medicine and Public Health, 3(3), 630-635. doi: 10.18203/2394-6040.ijcmph20160623

634 Liukkonen, J., Barkoukis, V., Watt, A., \& Jaakola, T. (2010). The relationship between motivational climate and students' emotional experiences and effort in school physical education. Journal of Educational Research, 103, 1-14. doi: 10.1080/00220670903383044

Moreno-Murcia, J. A., Hernandez, E. H., Pedreno, N. B., \& Neipp, M. C. (2017). Understanding contextual relation in promotion of physical exercise from autonomy support. International Journal of Psychological Studies, 9, 1-9. doi: 10.5539/ijps.v9n1p1

MacCallum, R. C., Browne, M. W., \& Sugawara, H. M. (1996). Power analysis and determination of sample size for covariance structure modeling. Psychological Methods, 1, 130-149. doi: 10.1037/1082-989X.1.2.130.

McCarney, R., Warner, J., lliffe, S., Van Haselen, R., Griffin, M., \& Fisher, P. (2007). The Hawthorne Effect: A randomised, controlled trial. BMC Medical Research Methodology, 7(1), 30. doi: $10.1186 / 1471-2288-7-30$

Mandigo, J., Holt, N., Anderson, A., \& Sheppard, J. (2008). Children's motivational experiences following autonomy-supportive games lessons. European Physical Education Review, 14, 407425. doi:10.1177/1356336X08095673

Marsh, H. W., Hau, K. T., \& Wen, Z. (2004). In search of golden rules: Comment on hypothesis-testing approaches to setting cutoff values for fit indexes and dangers in overgeneralizing $\mathrm{Hu}$ and

McLachlan, S., \& Hagger, M. S. (2010). Effects of an autonomy-supportive intervention on tutor 654 behaviors in a higher education context. Teaching and Teacher Education, 26, 1205-1211. doi:10.1016/j.tate.2010.01.006 
656 Mouratidis, A. A., Vansteenkiste, M., Sideridis, G., \& Lens, W. (2011). Vitality and interest-enjoyment as a function of class-to-class variation in need-supportive teaching and pupils' autonomous motivation. Journal of Educational Psychology, 103, 353. doi: 10.1037/a0022773

Mullen, E., Markland, D. A., \& Ingledew, D. K. (1997). A graded conceptualisation of self-determination in the regulation of exercise behaviour: Development of a measure using confirmatory factor analysis. Personality and Individual Differences, 23, 745-752. doi: 10.1016/S0191-

662 8869(97)00107-4

Murcia, J. A. M., Lacarcel, J. A. V., \& Alvarez, F. D. (2010). Search for autonomy in motor task learning in physical education university students. European Journal of Psychology of Education, 25, 3747. doi:10.1007/s10212-009-0008-7

Ntovolis, Y., Barkoukis, V., Michelinakis, E., \& Tsorbatzoudis, H. (2015). An application of the transcontextual model of motivation in elementary school physical education. Physical Educator, 72, 123-141. doi: 10.18666/TPE-2015-V72-I5-5111

Olson, J. L., Ireland, M. J., March, S., Biddle, S. J. H., \& Hagger, M. S. (2019). Physical activity in periurban communities: Testing intentional and implicit processes within an ecological framework. Applied Psychology: Health and Well-Being. doi: 10.1111/aphw.12182

Polet, J., Hassandra, M., Lintunen, T., Laukkanen, A., Hankonen, N., Hirvensalo, M., .. Hagger, M. S. (2019). Using physical education to promote out-of school physical activity in lower secondary school students - A randomized controlled trial protocol. BMC Public Health, 19, 157. doi:

676 Perlman, D. (2010). Change in affect and needs satisfaction for amotivated students within the sport education model. Journal of Teaching in Physical Education, 29, 433-445. doi: 
679 Reeve, J. (2009). Why teachers adopt a controlling motivating style toward students and how they can become more autonomy supportive. Educational Psychologist, 44, 159-175. doi: $10.1080 / 00461520903028990$

Reeve J. (2016) Autonomy-Supportive Teaching: What It Is, How to Do It. In: Liu W., Wang J., Ryan R. (eds) Building Autonomous Learners (pp. 129-152). Springer, Singapore.

Reeve, J., \& Jang, H. (2006). What teachers say and do to support students' autonomy during a 685 learning activity. Journal of Educational Psychology, 98, 209-218. doi: 10.1037/00220663.98.1.209

Revelle, W. (2018). psych: Procedures for psychological, psychometric, and personality research. Retrieved May 6, 2018, from https://cran.r-project.org/web/packages/psych/index.html`

Revelle, W. (2019). Using R and the psych package to find $\omega$. Retrieved June 1, 2019, from http://personality-project.org/r/psych/HowTo/omega.pdf

Rosseel, Y. (2012). lavaan: An R package for structural equation modeling. Journal of Statistical

Ryan, R. M., \& Connell, J. P. (1989). Perceived locus of causality and internalization: Examining reasons for acting in two domains. Journal of Personality and Social Psychology, 57, 749-761. doi: 10.1037/0022-3514.57.5.749

Shen, B., McCaughtry, N., \& Martin, J. (2007). The influence of self-determination in physical education on leisure-time physical activity behavior. Research Quarterly for Exercise and Sport, 78, 328-

699 Shen, B., McCaughtry, N., \& Martin, J. (2008). Urban adolescents' exercise intentions and behaviors: An exploratory study of a trans-contextual model. Contemporary Educational Psychology, 33, 841-858. doi: 10.1016/j.cedpsych.2007.09.002 
Su, Y. L., \& Reeve, J. (2011). A meta-analysis of the effectiveness of intervention programs designed to support autonomy. Educational Psychology Review, 23, 159-188. doi:10.1007/s10648-0109142-9147.

Suchert, V., Hanewinkel, R., \& Isensee, B. (2015). Sedentary behavior and indicators of mental health in school-aged children and adolescents: A systematic review. Preventive Medicine, 76, 48-57. doi: 10.1016/j.ypmed.2015.03.026

Teixeira, P. J., Marques, M. M., Silva, M. N., Brunet, J., Duda, J., Haerens, L., . . Hagger, M. S. (2020). Classification of techniques used in self-determination theory-based interventions in health contexts: An expert consensus study. Motivation Science. doi: 10.1037/mot0000172

Tessier, D., Sarrazin, P., \& Ntoumanis, N. (2010). The effect of an intervention to improve newly qualified teachers' interpersonal style, students motivation and psychological need satisfaction in sport-based physical education. Contemporary Educational Psychology, 35(4), 242-253. doi: 10.1016/j.cedpsych.2010.05.005

Tsorbatzoudis, H., Grouios, G., Alexandris, K., \& Barkoukis, V. (2007). Physical Education and Education through Sport in Greece. In G. Klein \& K. Hardman (Eds), Physical Education and Sport Education in the European Union (pp. 162-171). Paris, Editions Revue EP.S.

Vallerand, R. J. (1997). Toward a hierarchical model of intrinsic and extrinsic motivation. In: M. Zanna (Ed.), Advances in Experimental Social Psychology, Vol. 29 (pp. 271-360). Toronto: Academic Press.

Vallerand, R.J. (2007). A hierarchical model of intrinsic and extrinsic motivation for sport and physical activity. In M.S. Hagger \& N.L.D. Chatzisarantis (Eds.), Intrinsic Motivation and SelfDetermination in Exercise and Sport (pp. 255-279). Champaign, IL: Human Kinetics.

Vallerand, R. J. \& Ratelle, C. F. (2002). Intrinsic and extrinsic motivation: A hierarchical model. In E.L University of Rochester Press. 
727 Zhang, Z., \& Yuan, K.-H. (Eds.). (2018). Practical statistical power analysis using Webpower and R. 728 Granger, IN: ISDSA Press.

729 


\begin{tabular}{|c|c|c|c|c|}
\hline October & November & December & January-March & April \\
\hline \multirow{2}{*}{$\begin{array}{c}\text { Teacher } \\
\text { Recruitment } \\
\text { and Random } \\
\text { Assignment into } \\
\text { Conditions }\end{array}$} & $\begin{array}{c}\text { Teacher } \\
\text { Education on } \\
\text { Autonomy } \\
\text { Sunportive }\end{array}$ & $\begin{array}{c}1^{\text {st }} \\
\text { measurement } \\
\text { occasion } \\
\text { Wave } 1\end{array}$ & Intervention & $\begin{array}{c}2^{\text {nd }} \\
\text { measurement } \\
\text { occasion } \\
\text { Wave } 1\end{array}$ \\
\hline & & $\begin{array}{c}1^{\text {st }} \\
\text { measurement } \\
\text { occasion } \\
\text { Wave } 2 \text { ( } 3 \\
\text { weeks after } \\
\text { Wave } 1 \text { ) }\end{array}$ & & $\begin{array}{c}\text { 2nd } \\
\text { measurement } \\
\text { occasion } \\
\text { Wave } 2 \text { ( } 3 \\
\text { weeks after } \\
\text { Wave } 1 \text { ) }\end{array}$ \\
\hline
\end{tabular}

731

732 Figure 1. Timeline of the study design and data collection. 


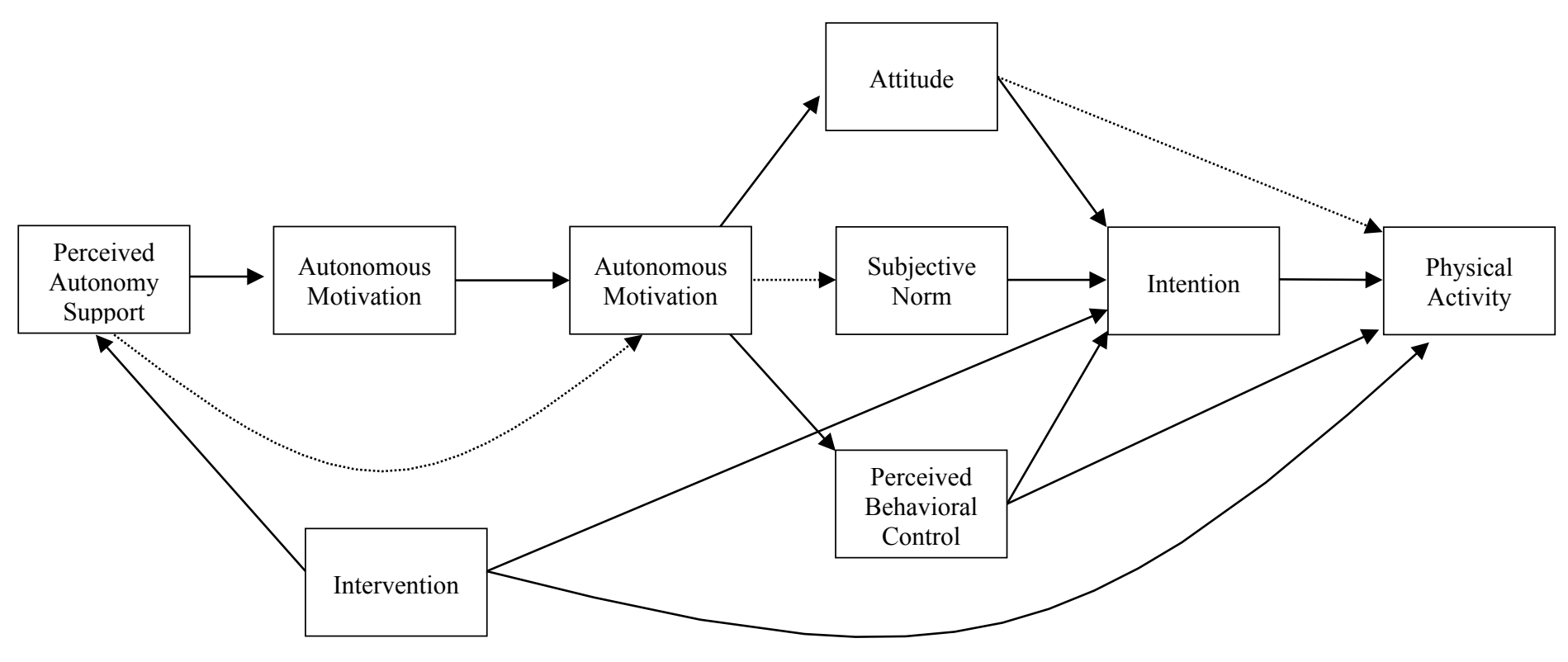

Figure 2. Proposed model illustrating effects of the trans-contextual model intervention on leisure-time physical activity and model constructs. Effects of gender, age, and past physical activity behavior as control variables on each variable in the model omitted for clarity. 


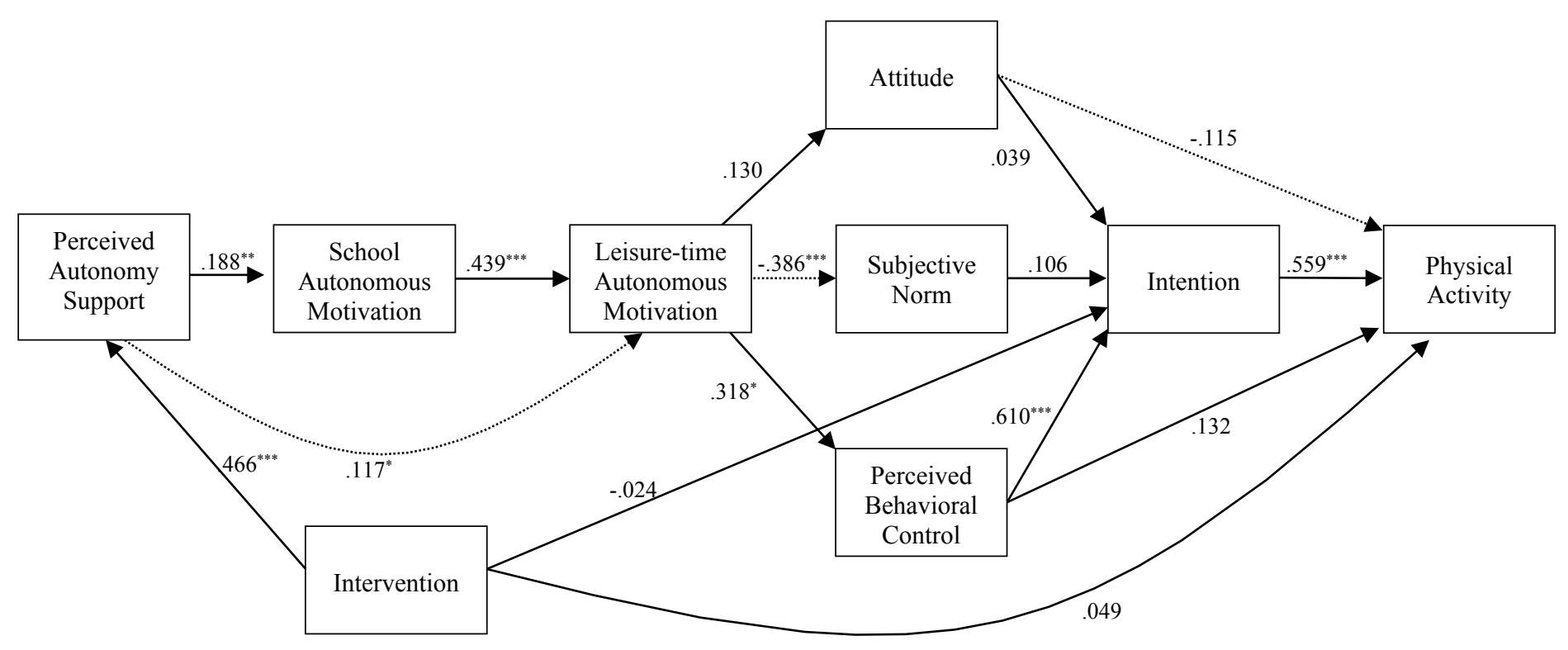


Figure 3. Standardized parameter estimates of effects in the path analytic model of the trans-contextual model intervention effects. Psychological variables (intention, attitude, subjective norm, perceived behavioral control, autonomous motivation in leisure time and school contexts, and perceived autonomy support) are residualized change scores derived from the regression of each variable at follow-up on itself at baseline. Statistically significant specific indirect effects not shown in model: Intervention $\rightarrow$ Perceived autonomy support $\rightarrow$ Autonomous motivation in PE $(\beta=.087, p=.003)$; Intervention $\rightarrow$ Perceived autonomy support $\rightarrow$ Autonomous motivation in leisure time $(\beta=.077, p=.004)$; Perceived autonomy support $\rightarrow$ Autonomous motivation in $P E \rightarrow$ Autonomous motivation in leisure time $(\beta=.082, p=.004)$; Autonomous motivation in leisure time $\rightarrow$ Perceived behavioral control $\rightarrow$ Intention $(\beta=.194, p=.036)$; Perceived behavioral control $\rightarrow$ Intention $\rightarrow$ Physical activity behavior $(\beta=.342, p<.001)$; Autonomous motivation in leisure time $\rightarrow$ Perceived behavioral control $\rightarrow$ Intention $\rightarrow$ Physical activity behavior $(\beta=.109, p=.048) ;$. Statistically significant total effects: Perceived autonomy support $\rightarrow$ Autonomous motivation in leisure time $(\beta=.199, p$ $=.004)$; Intervention $\rightarrow$ Autonomous motivation in leisure time $(\beta=.093, p=.004)$; Perceived behavioral control $\rightarrow$ Physical activity behavior $(\beta=.474, p<.001)$. Effects of gender, age, and past physical activity behavior as control variables on each variable in the model omitted for clarity, paths freely estimated in the model but not depicted in diagram: Gender $\rightarrow$ Perceived autonomy support $(\beta=.022, p=.712)$; Gender $\rightarrow$ Autonomous motivation in $P E(\beta=.185, p=.005)$; Gender $\rightarrow$ Autonomous motivation in leisure time $(\beta=.013, p=.838)$; Gender $\rightarrow$ Attitude $(\beta=-.003, p=.968)$; Gender $\rightarrow$ Subjective norms $(\beta=-.153, p=.021)$; Gender $\rightarrow$ Perceived behavioral control $(\beta=-.131, p=.052)$; Gender $\rightarrow$ Intention $(\beta=.005, p=.929)$; Gender $\rightarrow$ Physical activity $(\beta=-.070, p=.171)$;

Age $\rightarrow$ Perceived autonomy support $(\beta=.166, p=.013)$; Age $\rightarrow$ Autonomous motivation in $\mathrm{PE}(\beta=.082, p=.174)$; Age $\rightarrow$ Autonomous motivation in leisure time $(\beta=.080, p=.157) ;$ Age $\rightarrow$ Attitude $(\beta=-.048, p=.534) ;$ Age $\rightarrow$ Subjective norms $(\beta=-.081, p=.188) ;$ Age $\rightarrow$ Perceived behavioral control $(\beta=-.055, p=.469) ;$ Age $\rightarrow$ Intention $(\beta=.005, p=.929) ;$ Age $\rightarrow$ Physical activity $(\beta=.058, p=222)$; Past physical activity behavior $\rightarrow$ Perceived autonomy support $(\beta=-.003, p$ $=.962)$; Past physical activity behavior $\rightarrow$ Autonomous motivation in $\mathrm{PE}(\beta=.052, p=.450)$; Past physical activity behavior $\rightarrow$ Autonomous motivation in leisure time $(\beta=-.008, p=.885)$; Past physical activity behavior $\rightarrow$ Attitude $(\beta=.023, p=.767)$; Past physical activity behavior $\rightarrow$ Subjective norms $(\beta=.098, p=.098)$; Past physical activity behavior $\rightarrow$ Perceived behavioral control $(\beta=.027, p=.700)$; Past physical activity behavior $\rightarrow$ Intention $(\beta=.053, p=.300) ;$ Past physical activity behavior $\rightarrow$ Physical activity $(\beta=.122, p=.018)$.

${ }^{*} p<.05{ }^{* *} p<.01{ }^{* * *} p<.001$ 
TRANS-CONTEXTUAL MODEL INTERVENTION

Table 1

Example Content of Autonomy-Supportive Strategies

Theme and focus $\quad$ Example content

\begin{tabular}{ll}
$\begin{array}{l}\text { Student motivation } \\
\text { and autonomy } \\
\text { support }\end{array}$ & $\begin{array}{l}\text { Offering choices to students on various aspects of the lesson. Allowing students work } \\
\text { determine the pace of the lesson. Providing rationale for the structure of the lessons } \\
\text { and the choice of exercises. }\end{array}$ \\
$\begin{array}{l}\text { Developing social } \\
\text { interactions }\end{array}$ & $\begin{array}{l}\text { Opportunities for students to work in small groups. Opportunities for students to work } \\
\text { in multiple groups. Reciprocal teaching style. }\end{array}$ \\
$\begin{array}{l}\text { Recognition and } \\
\text { praise }\end{array}$ & $\begin{array}{l}\text { Recognition to all students. Recognition of motor performance as well as effort and } \\
\text { interest towards the lesson. }\end{array}$ \\
$\begin{array}{l}\text { Developing a sense } \\
\text { of competence }\end{array}$ & $\begin{array}{l}\text { Emphasis on personal development. Opportunities for students to work on their own } \\
\text { pace. Goal setting. Inclusion teaching style. }\end{array}$ \\
$\begin{array}{l}\text { Avoiding control: } \\
\text { Maintaining } \\
\text { autonomy support }\end{array}$ & $\begin{array}{l}\text { Avoiding exhibiting solutions/answers, monopolizing learning materials, uttering } \\
\text { solutions/answers, setting deadlines, uttering directives/commands, asking controlling } \\
\text { questions, emphasizing students' obligations, using judgmental language and } \\
\text { criticizing the students }\end{array}$ \\
\hline
\end{tabular}


TRANS-CONTEXTUAL MODEL INTERVENTION

Table 2

Zero-Order Intercorrelations and Reliability Coefficients for Study Variables

\begin{tabular}{|c|c|c|c|c|c|c|c|c|c|c|c|c|c|c|c|}
\hline Variable & $\omega^{\mathrm{a}}$ & $M^{b}$ & $S D^{\mathrm{b}}$ & 1 & 2 & 3 & 4 & 5 & 6 & 7 & 8 & 9 & 10 & 11 & 12 \\
\hline \multirow[t]{2}{*}{ 1. Perceived autonomy support } & .931 & 4.691 & 1.056 & - & & & & & & & & & & & \\
\hline & .931 & 5.268 & 0.915 & & & & & & & & & & & & \\
\hline \multirow[t]{2}{*}{ 2. Autonomous motivation (PE) } & $.583^{c}$ & 2.016 & 2.413 & $.179^{* *}$ & - & & & & & & & & & & \\
\hline & $.508^{\mathrm{c}}$ & 2.052 & 2.417 & & & & & & & & & & & & \\
\hline \multirow[t]{2}{*}{ 3. Autonomous motivation (LT) } & $.830^{\mathrm{d}}$ & 7.538 & 4.642 & $.235^{* *}$ & $.484^{\star * \star}$ & - & & & & & & & & & \\
\hline & .900 & 6.947 & 5.111 & & & & & & & & & & & & \\
\hline \multirow[t]{2}{*}{ 4. Attitude } & .878 & 4.803 & 0.966 & $.165^{*}$ & .126 & .175 & - & & & & & & & & \\
\hline & .867 & 4.908 & 0.873 & & & & & & & & & & & & \\
\hline \multirow[t]{2}{*}{ 5. Subjective norm } & $.484^{\mathrm{c}}$ & 4.020 & 1.517 & $.161^{* *}$ & -.087 & $-.379^{* * *}$ & $.129^{*}$ & - & & & & & & & \\
\hline & $.699^{c}$ & 4.048 & 1.720 & & & & & & & & & & & & \\
\hline \multirow[t]{2}{*}{ 6. Perceived behavioral control } & .802 & 5.381 & 1.238 & $.447^{\star \star \star}$ & $.208^{* *}$ & $.236^{* *}$ & $.331^{* \star *}$ & $.241^{* \star *}$ & - & & & & & & \\
\hline & .823 & 5.475 & 1.235 & & & & & & & & & & & & \\
\hline \multirow[t]{2}{*}{ 7. Intention } & .809 & 4.720 & 1.463 & $.269^{* * *}$ & $.214^{*}$ & $.221^{* *}$ & $.238^{* \star *}$ & $.244^{* \star *}$ & $.658^{* \star *}$ & - & & & & & \\
\hline & .812 & 4.762 & 1.435 & & & & & & & & & & & & \\
\hline 8. Physical activity behavior & - & 3.996 & 1.431 & $.132^{*}$ & $.277^{\star \star \star}$ & $.173^{*}$ & .116 & $.215^{* * *}$ & $.487^{\star \star \star}$ & $.642^{* \star *}$ & - & & & & \\
\hline 9. Past physical activity behavior & - & 4.025 & 1.414 & .000 & .055 & .019 & .034 & .097 & .070 & .089 & $.197^{\star *}$ & - & & & \\
\hline 10. Gender ${ }^{e}$ & - & - & - & -.032 & $.173^{*}$ & $.157^{*}$ & .015 & $-.224^{* * *}$ & -.046 & -.031 & -.113 & -.103 & - & & \\
\hline 11. Age & - & 13.432 & 0.825 & .048 & .080 & .049 & -.012 & .081 & -.018 & .034 & .050 & -.041 & -.120 & - & \\
\hline 12. Intervention ${ }^{f}$ & - & - & - & $.437^{* * * *}$ & $.191^{* *}$ & $.176^{* *}$ & .091 & -.105 & $.196^{*}$ & .073 & .126 & .116 & -.038 & $-.204^{* * *}$ & - \\
\hline
\end{tabular}

Note. $\mathrm{PE}=$ Physical education; $\mathrm{LT}=$ Leisure-time physical activity. Correlations among psychological constructs are for residualized change scores derived from the regression of the construct at post intervention follow-up on its baseline score. ${ }^{a}$ Revelle's (2019) Omega $(\omega)$ reliability coefficient, upper values are for scales at baseline and lower values are for post-intervention follow-up; bescriptive statistics are for averaged scales at each time point, upper values are for scales at baseline and lower values are for post-intervention follow-up; 'Two item scale so reliability is Spearman-Brown inter-item correlation; dMatrix for calculation of $\omega$ reliability coefficient was not positive definite,

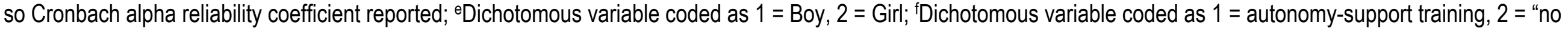
training" control. ${ }^{*} p<.05^{* *} p<.01^{* * *} p<.001$ 
